\title{
In There any Connection between Normal Blood Pressure and Watching Cricket?
}

\author{
Muhammad Imran Qadir and Arslan Hassan* \\ Institute of Molecular Biology and Biotechnology, Pakistan
}

*Corresponding author: Arslan Hassan, Institute of Molecular Biology and

Biotechnology, Bahauddin Zakariya University, Multan, Pakistan.

\begin{abstract}
The objective of our current study was to correlate interest in watching cricket and blood pressure. Blood pressure applies force on blood to move through our body and transport oxygen or nutrients to our tissues. The sphygmomanometer is a device that measure the pressure of blood on our arteries. It is measured in mmHg unit. It is measured in two figures - systolic and diastolic blood pressure. Cricket is a game which is famous worldwide. There is great difference in interests of people according to their thinking. Some people consider cricket a noble game and take interest in watching and playing. There are also people who do not like cricket and consider it a time wasting on even playing. There is also difference among people which are more interested and extremist for cricket. It is concluded from the current study that there is no scientific relationship between normal blood pressure and watching cricket [1]
\end{abstract}

Keywords: Blood pressure; Sphygmomanometer; Systolic and diastolic; Watching cricket

\section{Introduction}

Normal blood pressure is necessary for living of an individual. The pressure of blood applies force on blood to move through our body and transport oxygen or nutrients to our tissues. It is also important that it transport WBCs and antibodies and hormones to create the immunity of our body. Blood pressure may increase to much or decrease to very low both are dangerous situations for our body. The sphygmomanometer is a device that measure the pressure of blood on our arteries and consist of cuff, hand pump and electric meter [2]. Blood pressure always measure in unit of mmHg (millimeters of mercury). Blood pressure always measure in two figures - first value is high due to heart's contraction called systolic pressure and second is low value due to resting period of our heartbeats called diastolic pressure [3]. For example, normal blood pressure of adults is $120 \mathrm{mmHg}$ and $80 \mathrm{mmHg}$, systolic is higher than diastolic pressure. If blood pressure increases it increases the chances of hearts diseases and hypertension [4,5], failure of kidney and can cause the problems of eyes. It is related with age which may harden the blood arteries, so pressure is increased. If the blood pressure is very low to 80/50 then it will make you weak, lightheaded, and dim. The causes of low blood pressure may be dehydration (not drinking sufficient water), much loss of blood, or taking too much medicines. To maintain normal blood pressure is very necessary for our life so we must take some steps in our daily life routine to make blood pressure normal, always try to retain the fit weightiness, take exercise daily, eat well food, smoking and alcohol keep far away from body and try to manage tension. Higher blood pressure can cause the serious diseases and very dangerous for health so always try to maintain the normal blood pressure [6].

Cricket is a game which is famous worldwide and about 2.5 billion fans of cricket specially in Asia, Australia and UK. Cricket is played between two teams both have 11 players. There are three formats of cricket internationally T20, ODI and Test. There is great difference in interests of people according to their thinking. Some people consider cricket a noble game and take interest in watching and playing. There are also people who do not like cricket and consider it a time wasting on even playing. There is also difference among people which are more interested and extremist for cricket. According to their interest in games, many types of fans come into seeing [7]. Some are quite fans which seems quite from outside but cheering inside for teams. Some are loud fans which are always excited on every type of playing. Some are drunk fans which throw things and become uncontrollable on losing. Some people are allknowing fans which gives analysis before and after match and on every over of playing. Some are temporary fans which may left their teams when they are not playing well. Some are positive fans which 
love their teams in every situation. So, people have different types of interests about the same sport cricket [8].

The object of current study was to examine the connection between normal blood pressure of people and variation of interest among people for watching cricket [9].

\section{Material and Methods}

\section{Protocol to Examine the blood Pressure}

Firstly, we consent the subjects to calculate the pressure of blood on arteries. They gave us permission and we measure their blood pressure by sphygmomanometer one by one and inquired from subjects that they take interest in watching cricket or not and listed them by systolic pressure with people's interest in watching cricket and another list of diastolic pressure with interest of people in cricket [10]. Total of 190 subjects participated in the current study. All the subjects were the students of Bahauddin Zakariya University, Multan, Pakistan.

\section{Statistical analysis}

Statistical Analysis and T-test that were applied on values for result was made by Microsoft Excel.

\section{Results and Discussions}

Table 1 of systolic blood pressure, we calculated the average of blood pressure of all males and females separately. Then we calculated the standard deviation of all values of blood pressure of males and females and. Then we calculated the p- value in similar way. p-value of 0.05 is considered as standard value. Males which are interested in watching cricket have the average systolic blood pressure of 129 and standard deviation is 14 . Females which are interested in watching cricket have average systolic blood pressure is 118 and standard deviation of 13 . Both males and females have $p$ value higher than the standard value.

Table 1: Systolic blood pressure and variation of people's interest for watching cricket.

\begin{tabular}{|c|c|c|c|}
\hline Gender & $\begin{array}{c}\text { Interested in } \\
\text { Watching Cricket }\end{array}$ & $\begin{array}{c}\text { Non-Interested in } \\
\text { Watching Cricket }\end{array}$ & p Value \\
\hline Males & $129 \pm 14$ & $125 \pm 13$ & 0.63 \\
\hline Females & $118 \pm 13$ & $113 \pm 13$ & 0.06 \\
\hline
\end{tabular}

$p<0.05$ hence relation of between them is non-significant.

Table 2 of diastolic blood pressure, we calculated the average of diastolic blood pressure of males, females and combine. Males those are interested in cricket have average diastolic blood pressure 75 and standard deviation 13. Females those are interested in cricket have average diastolic blood pressure 74 and standard deviation of
11. Males those are not interested in watching cricket have average diastolic blood pressure is 62 with SD of 12 and females with average blood pressure those are not interested in watching cricket is 75 with SD of 13. P - value of males and females is higher than 0.05 . So, the relation between the average diastolic blood pressure and people's interest in watching cricket is non-significant.

Table 2: Diastolic Blood pressure and variations of interest among people for watching cricket

\begin{tabular}{|c|c|c|c|}
\hline Gender & Interested in Cricket & Non-Interested & P Value \\
\hline For Males & $75 \pm 13$ & $62 \pm 12$ & 0.19 \\
\hline For Females & $74 \pm 11$ & $75 \pm 13$ & 0.69 \\
\hline
\end{tabular}

$p<0.05$ hence relation of between them is non-significant.

\section{Conclusion}

It is concluded from the current study that there is no scientific relationship between normal blood pressure and watching cricket.

\section{Acknowledgement}

None.

\section{Conflict of Interest}

No conflict of interest.

\section{References}

1. Samariya D, Matariya A, Raval D, Babu LD, Raj ED, et al. (2016) A Hybrid Approach for Big Data Analysis of Cricket Fan Sentiments in Twitter. In Proceedings of International Conference on ICT for Sustainable Development Pp. 503-512.

2. Lamego M, Kiani MJ, Lam K, Dalvi C, Vo H, et al. (2017) Cercacor Labs Inc, assignee. Blood pressure measurement method.

3. Qadir MI, Javid A (2018) Awareness about Crohn's Disease in biotechnology students. GloAdv Res J Med Medical Sci 7(3): 062-064.

4. Qadir MI, Saleem A (2018) Awareness about ischemic heart disease in university biotechnology students. GloAdv Res J Med Medical Sci 7(3): 059-061.

5. Qadir MI, Ishfaq S (2018) Awareness about hypertension in biology students. Int J Mod Pharma Res 7(2): 08-10.

6. Qadir MI, Mehwish (2018) Awareness about psoriasis disease. Int J Mod Pharma Res 7(2): 17-18.

7. Qadir MI, Shahzad R (2018) Awareness about obesity in postgraduate students of biotechnology. Int J Mod Pharma Res 7(2): 14-16.

8. Qadir MI, Rizvi M (2018) Awareness about thalassemia in post graduate students. MOJ Lymphology \& Phlebology 2(1): 14-16.

9. Qadir MI, Ghalia BA (2018) Awareness survey about colorectal cancer in students of M. Phil Biotechnology at Bahauddin Zakariya University, Multan, Pakistan. Nov Appro in Can Study 1(3): NACS.000514.2018.

10. Qadir MI, Saba G (2018) Awareness about intestinal cancer in university student. Nov Appro in Can Study 1(3): NACS.000515.2018. 